\title{
Mode Conversion and Anomalous Transport in Kelvin-Helmholtz Vortices and Kinetic Alfvén Waves at the Earth's Magnetopause
}

\author{
C. C. Chaston, ${ }^{1}$ M. Wilber, ${ }^{1}$ F. S. Mozer, ${ }^{1}$ M. Fujimoto, ${ }^{2}$ M. L. Goldstein, ${ }^{3}$ M. Acuna, ${ }^{3}$ H. Reme, ${ }^{4}$ and A. Fazakerley ${ }^{5}$ \\ ${ }^{1}$ Space Science Laboratory, University of California, Berkeley, California, USA \\ ${ }^{2}$ ISAS/JAXA, Space Plasma Division, Kanagawa, Japan \\ ${ }^{3}$ NASA Goddard Space Flight Center, Greenbelt, Maryland, USA \\ ${ }^{4}$ CESR, Toulouse, France \\ ${ }^{5}$ Mullard Space Science Laboratory, Dorking, United Kingdom
}

(Received 26 April 2007; published 26 October 2007)

\begin{abstract}
Observations at the Earth's magnetopause identify mode conversion from surface to kinetic Alfvén waves at the Alfvén resonance. Kinetic Alfvén waves radiate into the magnetosphere from the resonance with parallel scales up to the order of the geomagnetic field-line length and spectral energy densities obeying a $k_{\perp}^{-2.4}$ power law. Amplitudes at the Alfvén resonance are sufficient to both demagnetize ions across the magnetopause and provide field-aligned electron bursts. These waves provide diffusive transport across the magnetopause sufficient for boundary layer formation.
\end{abstract}

DOI: 10.1103/PhysRevLett.99.175004

The outer boundary of the Earth's magnetic field known as the magnetopause blocks the entry of shocked solarwind plasma into the magnetosphere. However, observations show the existence inside the magnetopause of a boundary layer of these plasmas with width an order of magnitude larger than an ion gyro-radii [1]. These observations are evidence for the transport of solar-wind plasmas across the magnetopause [2]. While magnetic reconnection is generally considered to be the prime means through which this transport occurs, conditions in the solar wind that favor reconnection are not always present, yet boundary layers are still observed [3]. In this Letter we show that mode conversion [4] from surface mode Alfvén waves to kinetic Alfvén waves (KAWs) [5] across the magnetopause transports large electromagnetic energy fluxes across geomagnetic field lines from magnetosheath flows into the magnetosphere and provides spectral energy densities sufficient to drive anomalous transport at the rate required to account for boundary layer formation [6-9]. These results are relevant not only to the Earth's magnetosphere but also for understanding transport processes in solar flares and accretion disks [10,11] and destructive transport in tokamak and fusion plasmas [12].

Figure 1 shows observations at the magnetopause from the Cluster-4 spacecraft [13] during an extended period of northward pointing interplanetary magnetic field. At this time the spacecraft was located on the duskside flanks of the Earth's magnetosphere and traveling sunward. Figure 1(a) shows the magnetic field in the $Z$ direction of the geocentric solar magnetospheric coordinate system (GSM) [14]. It was demonstrated in a previous study [15] that the step-like field variations observed in the magnetic field are consistent with the traversal of the spacecraft through a series of anti-sunward propagating surface wave vortices driven by a Kelvin-Helmholtz $(\mathrm{KH})$ instability. The steep gradients indicated by the dashed blue lines in Fig. 1(a) show the transition of the spacecraft from
PACS numbers: 94.30.ch, 94.05.Lk, 94.05.Pt, 94.30.cs

the magnetosphere into the magnetosheath through the trailing edge of each vortex. Notably, at each of these transitions we find sharp oscillatory enhancements in the electric field as shown in Fig. 1(b). These oscillations extend into the magnetosphere. Plasma transport across the magnetopause is proven from CIS-HIA measurements

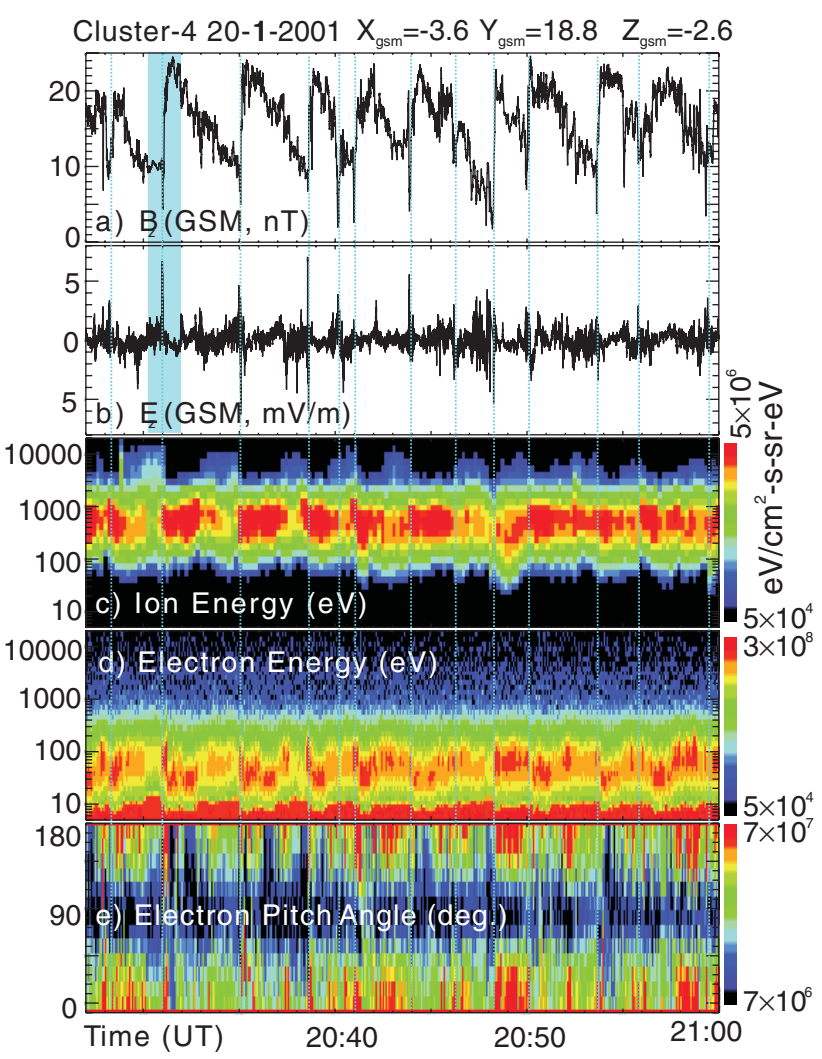

FIG. 1 (color). (a) and (b) $Z_{\mathrm{GSM}}$ magnetic and electric field, respectively. (c) Ion energy and pitch angle spectrograms from the CIS-HIA instrument on Cluster-3. (e) and (f) electron energy and pitch angle spectrograms from PEACE on Cluster-4. 
on the Cluster-3 spacecraft, shown in Fig. 1(c), which identify magnetosheath ions (centered on $\sim 400 \mathrm{eV}$ ) throughout the interval, and the superposition of the more energetic magnetospheric ions visible above $2 \mathrm{keV}$ to the left of each of the dashed blue lines when within the magnetosphere. Analysis of these data shows $T_{\perp} / T_{\|}>1$ [16] for the magnetosheath ions across the boundary with magnetospheric ions measured by the CIS-CODIF instrument on Cluster-4 containing significant densities of $\mathrm{O}^{+}$. Figures 1(d) and 1(e) indicate that throughout the interval shown we observe bursty field-aligned electrons $\left(0^{\circ}, 180^{\circ}\right)$ with the largest energies and fluxes found when the spacecraft is within the magnetosphere. Similar findings have been reported in reconnection events near the subsolar [17] and high latitude magnetopause [18]. The observations we report here are, however, suggestive of anomalous crossfield particle transport and field-aligned electron acceleration in the electromagnetic fluctuations we observe with each magnetopause transition, without the operation of magnetic reconnection.

Figure 2 shows an expanded view of a magnetosphere to magnetosheath transition (light blue shaded interval in Fig. 1). A correlation analysis using the Cluster-4 spacecraft in magnetic field and spacecraft potential while transiting the magnetopause reveals that this boundary moves along the unit vector $[-0.8,-0.6,-0.05]$ in the GSM coordinate system at a speed of $\sim 95 \mathrm{kms}^{-1}$. The quantities shown in Fig. 2 are presented in the boundary normal coordinate system $(\mathrm{BN})$ defined by this motion where $x_{\mathrm{BN}}$ and $y_{\mathrm{BN}}$ lie in the plane of the magnetopause and point closest to the $Z_{\mathrm{GSM}}$ and $Y_{\mathrm{GSM}}$ directions, respectively, while $z_{\mathrm{BN}}$ points inwards normal to the magnetopause and in the direction of the boundary motion. Figure 2(a) shows that $B_{y}$ abruptly decreases as the spacecraft enters the magnetosheath. Associated with this transition is a decrease in the Alfvén speed $\left(V_{A}\right)$ shown by the red trace. The phase speed of a surface wave across this gradient in $V_{A}$ is estimated as $V_{s} \approx\left[\left(B_{\text {Msphere }}{ }^{2}+B_{\text {Msheath }}{ }^{2}\right) /\right.$ $\left.\mu_{o}\left(\rho_{\text {Msphere }}+\rho_{\text {Msheath }}\right)\right]^{1 / 2}$ [19] where $V_{S}$ is the surface wave phase speed, $B_{\text {Msphere }}$ and $B_{\text {Msheath }}$ are the magnetic field strengths on the magnetospheric and magnetosheath sides of the magnetopause transition, and $\rho_{\text {Msphere }}$ and $\rho_{\text {Msheath }}$ are the mass densities. Across the transition shown in Fig. 2 we find $V_{S} \approx 240-255 \mathrm{kms}^{-1}$. The location where this velocity matches the local Alfvén speed across the boundary is known as the Alfvén resonance (AR) and is shown in Fig. 2 by the green shaded band.

At the AR the $x$ component of the electric field shown in Fig. 2(b) contains a sharp peak. This peak is preceded by oscillatory fluctuations extending from the magnetosphere out to the AR on the magnetopause. Figure 2(c) shows that these electric field fluctuations provide a net Poynting flux $(S)$ into the magnetosphere which at the AR has a value comparable to the energy flux of the magnetosheath flow in the shear layer $\left(\sim 3 \times 10^{-5} \mathrm{~W} / \mathrm{m}^{2}\right)$. While not shown here, these waves also provide field-aligned Poynting fluxes

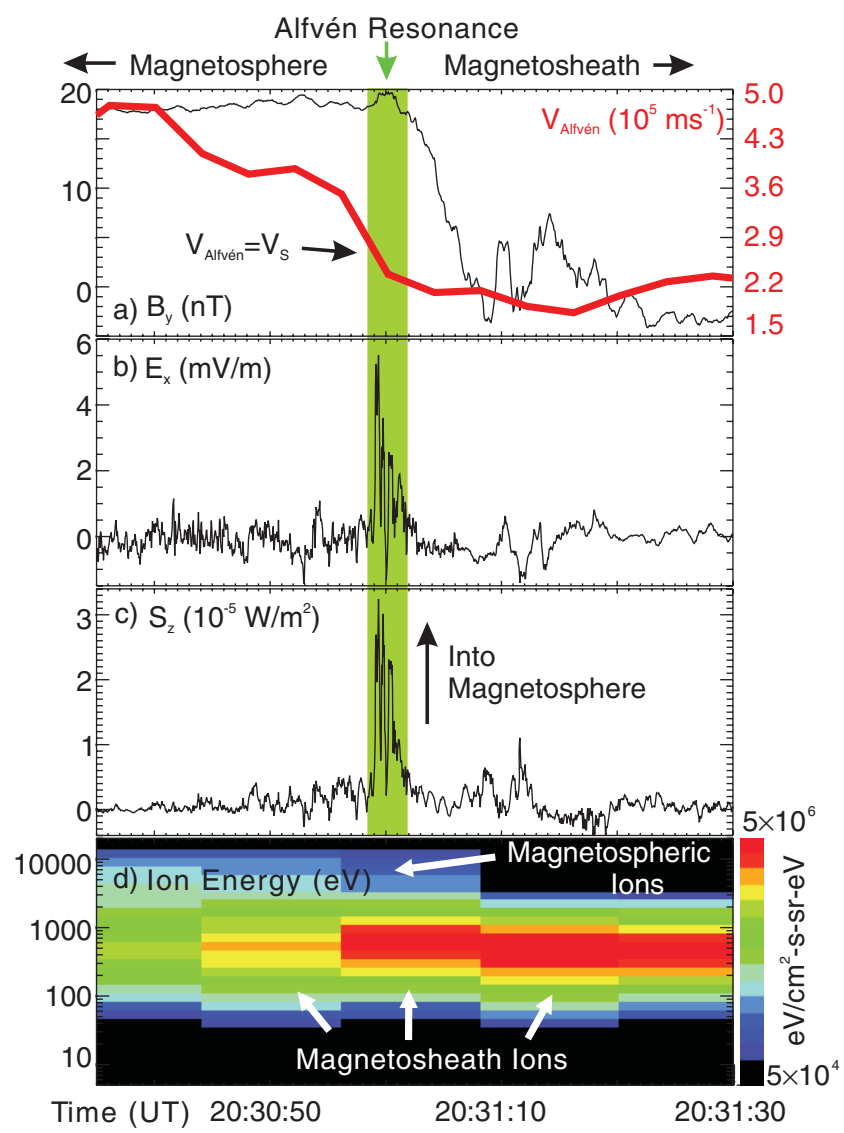

FIG. 2 (color). (a) $Y_{\mathrm{BN}}$-component magnetic field; the red trace shows the local Alfvén speed. (b) The $X_{\mathrm{BN}}$-component electric field. (c) The wave Poynting flux normal to the magnetopause boundary. (d) Ion energy spectrogram.

toward the ionosphere with a value mapped to $100 \mathrm{~km}$ of $\sim 50 \mathrm{~mW} / \mathrm{m}^{2}$ and sufficient to drive aurora. However, because the spacecraft trajectory is not normal to the magnetopause the variation of these fluxes with radial distance into the magnetosphere is not clear from these observations. Figure 2(d) shows that coincident with these waves we observe plasmas of mixed magnetospheric and magnetosheath composition inside the magnetosphere. Similar observations are made at every magnetopause transition over the interval where the Cluster spacecraft traverse the vortices. The amplitude of the electric field oscillations increases with increasing shear in the magnetic field across the magnetopause [20]. These observations suggest that the electromagnetic oscillations we observe are driven by mode conversion from the surface Alfvén waves or $\mathrm{KH}$ vortices at the AR on each magnetopause crossing and transport large fluxes of electromagnetic energy and plasma across field-lines from the magnetosheath into the magnetosphere.

To determine if we are observing KAWs radiating from the magnetopause, we compare the observed wave dispersion with that expected for KAWs. We assume that these waves are periodic transverse to the background magnetic 
field $\left(B_{o}\right)$ with vector potential $A_{\mu}(\nu, \mu, t)=A_{\mu}(\mu) \times$ $\exp [i(2 \pi \nu / \alpha-\omega t)]$ where $\nu$ and $\mu$ are the coordinates across and along $B_{o}$ respectively. $\alpha$ is the wave scale transverse to $B_{o}$ and is invariant along $\mu$. $\omega$ is the wave frequency. For values of electron plasma beta $\left(\beta_{e}\right)$ less than one and $\omega \ll \Omega_{i}$ the wave equation for Alfvén waves [21] is

$$
\begin{aligned}
\omega^{2} \lambda_{e}^{2} D^{2} A_{\mu}-\nabla_{\mu} V_{A}^{2} \chi \frac{h_{\nu}}{h_{\phi}} \nabla_{\mu} \frac{h_{\phi}}{h_{\nu}} A_{\mu} \\
+\frac{\rho_{s}{ }^{2} V_{A}{ }^{2}}{T_{e}} \nabla_{\mu} \frac{T_{e}}{h_{\nu} h_{\phi}} \nabla_{\mu} h_{\nu} h_{\phi} D^{2} A_{\mu}-\omega^{2} A_{\mu}=0
\end{aligned}
$$

where $D=i 2 \pi /\left(\alpha h_{\nu}\right)$, which locally provides $k_{\nu} \approx-i D$, and $\chi=-D^{2} \rho_{i}^{2}\left[1-I_{o}\left(-D^{2} \rho_{i}^{2}\right) \exp \left(D^{2} \rho_{i}^{2}\right)\right] . \quad \lambda_{e}=$ $c / \omega_{p e}$ is the electron inertial length, $T_{e}=m_{e} v_{e}{ }^{2}$ is the electron temperature, $\rho_{s}=v_{e} / \Omega_{i}$ is the ion acoustic gyroradius, $\rho_{i}$ is the ion gyroradius, and $I_{o}$ is the zeroth order modified Bessel function. $h_{\nu, \phi, \mu}$ are metrics which we derive numerically [22] for a Tsyganenko 2001 geomagnetic field model [23] using upstream parameters from the ACE spacecraft, and Dst from the World Data Center in Kyoto, Japan [24] $\left(n \approx 4 \mathrm{~cm}^{-3}, v \approx 400 \mathrm{kms}^{-1}\right.$, Dst $\approx$ $-28, B_{x \mathrm{gsm}} \approx-2.8 \mathrm{nT}, B_{y \mathrm{gsm}} \approx 1.0 \mathrm{nT}, B_{z \mathrm{gsm}} \approx 2.0 \mathrm{nT}$ ). These define a magnetic field model which provides field strengths very similar to those observed locally at Cluster. Equation (1) is then solved for eigenmodes in $A_{\mu}$ with boundary conditions defined by the high conductivity of the ionosphere at $100 \mathrm{~km}$ altitude where $E_{\nu}=-D \phi \rightarrow 0$ and $d B \phi / d \mu \rightarrow 0$. These quantities are given by

$$
\phi=\frac{V_{A}^{2}}{i \omega} \chi \frac{h_{\nu}}{h_{\phi}} \nabla_{\mu} \frac{h_{\phi}}{h_{\nu}} A_{\mu}
$$

and

$$
B_{\phi}=-D A_{\mu} .
$$

The wave carries a parallel electric field given by

$$
\begin{aligned}
E_{\|} & \approx E_{\mu} \\
& =\omega \lambda_{e}{ }^{2} D^{2} A_{\mu}+\frac{\rho_{s}{ }^{2} V_{A}{ }^{2}}{\omega T_{e}} \nabla_{\mu} \frac{T_{e}}{h_{\nu} h_{\phi}} \nabla_{\mu} h_{\nu} h_{\phi} D^{2} A_{\mu}
\end{aligned}
$$

which drives field-aligned electron acceleration and facilitates anomalous plasma transport. The plasma model along the geomagnetic field required for the solution of (1) is based on the averaged locally observed parameters $\left(n \approx 2.7 \mathrm{~cm}^{-3}, T_{i} \approx 160 \mathrm{eV}, T_{e} \approx 30 \mathrm{eV}\right)$ and observations made on similar field lines at different altitudes in a former study [21]. This model provides $\beta_{e} \leq 0.1$ along the length of the field line as required for the validity of (1).

To compare the observed fields and those given by (1) requires measurement of $\alpha$ or $k_{\nu}$. Under the conditions observed at the magnetopause the component of flow transverse to $B_{o}$ is $\sim 100 \mathrm{kms}^{-1}$ and far exceeds the wave phase speed transverse to $B_{o}$ (or in $\nu$ ) of the kinetic Alfvén wave for all reasonable values of $\alpha$ or $k_{\nu}$. This allows us to invert the wave spectra in spacecraft frame frequency $\left(\omega_{\mathrm{sp}}\right)$ to wave number as $\omega_{\mathrm{sp}} \approx \mathbf{k}_{\nu} \cdot \mathbf{v}_{\mathrm{sp}}$ where $\mathbf{v}_{\mathrm{sp}}$ is the flow speed measured in the spacecraft frame. Using this approach we can obtain the $k_{\nu}$ spectra in $E_{\nu}$ $\left(E_{\perp}\right)$ and $B_{\phi}\left(B_{\perp}\right)$ and hence $E_{\nu} / B_{\phi}\left(k_{\nu}\right)$. Averaged results using this analysis from the multiple magnetopause crossings observed from 19:50:00-21:00:00 UT are shown in Fig. 3(a) and reveal wave scales extending through $\rho_{i}, \rho_{s}$ and down to $\lambda_{e}$. The magnetic field oscillations vary as $\sim k_{\nu}^{-1.8(\sim-5 / 3)}$ and $\sim k_{\nu}^{-2.4(\sim-7 / 3)}$ with the break point at $\sim k_{\nu} \rho_{i} \approx 1$. Figure 3(b) shows the observed (black) and predicted $E_{\nu} / B_{\phi}\left(k_{\nu}\right)$ ratio from solution of 1 for the fundamental and first, second, and third harmonics (red) while the blue trace shows the local result obtained from the Fourier transform of (1) along $B_{o}\left(k_{\nu} \rightarrow k_{\perp}\right.$ and $\nabla \mu \rightarrow$ $i k_{\|}$so locally $E_{\perp} / B_{\perp} \approx V_{A}\left[1+k_{\perp}^{2} \rho_{i}^{2}\right] /\left[1+k_{\perp}{ }^{2} \rho_{i}{ }^{2}+\right.$ $\left.k_{\perp}^{2} \rho_{s}^{2}\right]^{1 / 2}$ ). These model results are evaluated at the position of the Cluster spacecraft in the Tsyganenko field model. The model results show that over most of the

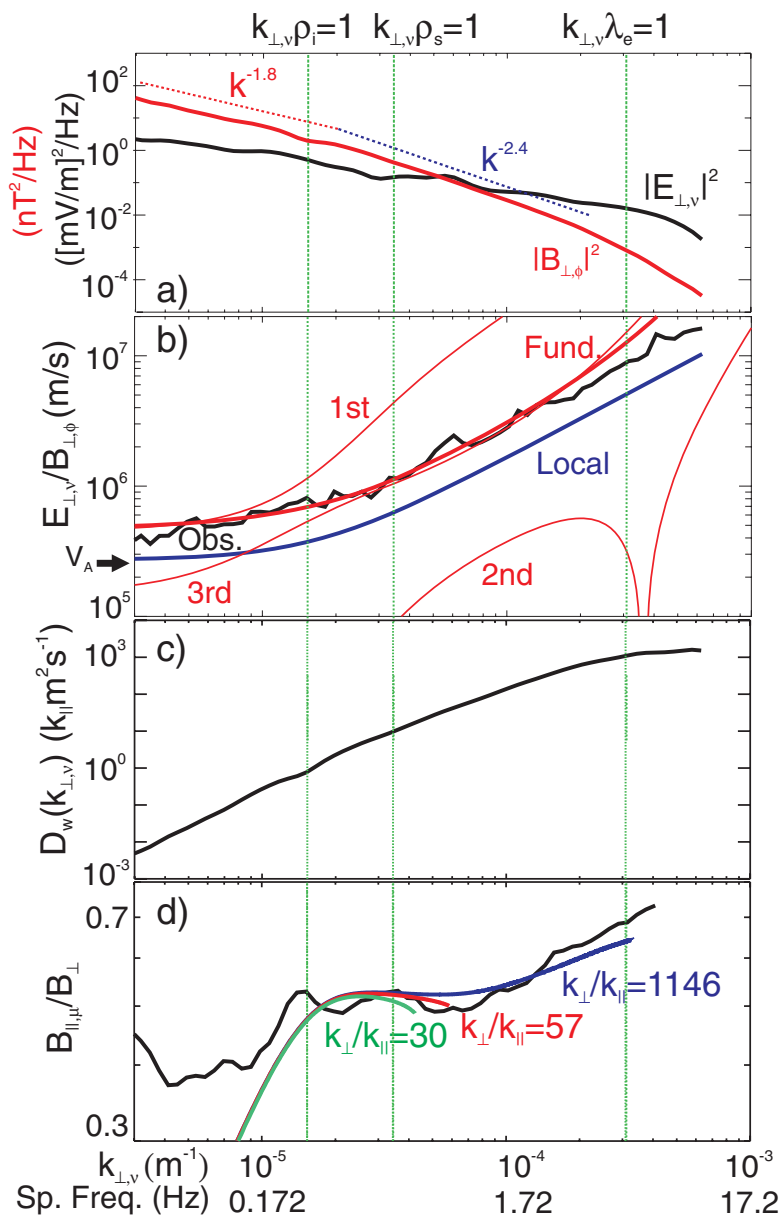

FIG. 3 (color). (a) Averaged spectral energy density in $E_{\perp, \nu}$ (black) and $B_{\perp, \phi}$ (red). (b) Averaged $E_{\perp, \nu} / B_{\perp, \phi}$ spectra from observations (black), local wave dispersion (blue) and nonlocal solutions (red) for the fundamental through to third harmonic. (c) Diffusion coefficient at each $k_{\perp, \nu}$. (d) Averaged ratio of compressive to transverse magnetic field amplitudes (black) and that given by local wave dispersion for $k_{\perp} / k_{\|}=30,57,1146$. 
observed range in $k_{\nu}$ the fundamental provides an excellent fit to the observed wave fields with the first and third harmonics providing good approximations to the data at small and large $k_{\nu}$ respectively. The wave frequency at $k_{\nu} \rho_{i}=1$ for the fundamental, first, and third harmonics are, respectively, 0.0012, 0.0021 , and $0.0042 \mathrm{~Hz}$. The local approximation also provides a reasonable estimate of the trend in $E_{\nu} / B_{\phi}\left(k_{\nu}\right)$ particularly at $k_{\nu} \rho_{i}>1$. The results demonstrate that these field fluctuations have the properties of KAWs with long parallel wavelengths. Furthermore, from (3) and for observed transverse wave amplitudes these waves carry parallel electric fields up to a few $\mu \mathrm{V} / \mathrm{m}$ at $k_{\nu} \rho_{i}=1$ to provide field-aligned potentials $\leq 100 \mathrm{~V}$. This is sufficient to account for the energies of the bursty field-aligned electron fluxes identified in Fig. 1.

Transport across the magnetopause in these waves can be estimated using the local quasilinear calculation [6] where the diffusion coefficient for a plasma where $V_{A} \sim$ $v_{i}\left(v_{i}=\right.$ ion thermal speed $)$, as found in this case, is given by

$D_{W}=\left(\frac{\pi}{8}\right)^{1 / 2} \sum_{k}\left(\frac{T_{e}}{T_{i}} k_{\perp}^{2} \rho_{i}^{2}\right)^{2} \frac{V_{A}^{2}}{k_{\| \mid} v_{i}}\left(\frac{\left|B_{\perp}(k)\right|}{B_{o}}\right)^{2} \exp \left(\frac{-V_{A}^{2}}{2 v_{i}^{2}}\right)$.

Substituting the observed plasma parameters into (4) we obtain the values shown in Fig. 3(c) which shows that waves between $k_{\perp} \rho_{i}=1$ and $k_{\perp} \lambda_{e}=1$ will provide the largest contribution to transport. The appropriate $k_{\|}$for each $k_{\perp}$ over this range is given by fitting the full local hot plasma dispersion relation to the observed ratio of compressive to transverse magnetic field oscillations as shown in Fig. 3(d). Good fits for $k_{\perp, \nu} \rho_{i} \geq 1$ are obtained for $k_{\perp} / k_{\|} \geq 30.0$ which at $k_{\perp, \nu} \rho_{i}=1$ provides wavelengths along $B_{o}$ of $\geq 1$ Earth radii and up to the fieldline length consistent with the eigenmodes solutions. Performing the sum in (4) for these $k_{\perp} / k_{\|}$we find the total diffusion coefficient for ions is $2.1 \times 10^{9} \leq D_{W} \leq 3.0 \times$ $10^{10} \mathrm{~m}^{2} \mathrm{~s}^{-1}$, similar to previous estimates where a single wave at $k_{\perp} \rho_{i}=1$ was assumed $[8,9,25]$. We note that the upper limit is probably not achieved due to the local approximation in (4) and because the Bohm diffusion coefficient based on the magnetosheath ion temperature has a maximum value over this interval of $\sim 3.3 \times$ $10^{9} \mathrm{~m}^{2} \mathrm{~s}^{-1}$. Using the density gradient at each magnetopause traversal determined from a cross correlation analysis between the Cluster- 4 spacecraft, the lower limit for the average flux across the magnetopause is $D_{w} \nabla_{z} n \approx$ $10^{10} \mathrm{~m}^{-2} \mathrm{~s}^{-1}$. This is sufficient to account for the nominal width of the low latitude boundary layer [7].

These observations show the operation of mode conversion from surface waves to KAWs on the magnetopause at the AR and the cross-field transport of large electromagnetic energy fluxes into the magnetosphere. The KAWs have transverse scales extending through $\rho_{i}, \rho_{s}$ and $\lambda_{e}$ and parallel scales at $k_{\perp} \rho_{i} \approx 1$ up to the field-line length. These waves carry parallel electric fields of sufficient amplitude to account for the observed field-aligned electron bursts. At the largest parallel scales these waves are field-line resonances. The KAW spectra can provide diffusion of magnetosheath plasma across the magnetopause at a rate sufficient to account for the nominal width of the low latitude boundary layer reported in several studies. As a final note, the electric field spikes observed at the AR, for the observed transverse scales, have amplitudes which exceed that required to demagnetize the ions across the magnetopause $\left(E_{\text {demag }} \approx B_{o} \Omega_{i} / k_{\perp} \approx 2 \mathrm{mV} / \mathrm{m}\right.$ at $k_{\perp} \rho_{i} \approx 1$ [26]). It is expected that this will lead to stochastic transverse ion energization [27] to account for $T_{\perp} / T_{\|}>1$ and enhance the transport process across the magnetopause.

This research was supported by NSF Grant No. ATM0602728 and No. ATM-0612614 and NASA Grant No. NNG05GL27G-06/08. We thank the ACE MAG and SWEPAM team, the ACE Science Center, and the WDC in Kyoto Japan.

[1] T. D. Phan and G. Paschmann, J. Geophys. Res. 101, 7801 (1996).

[2] G. Paschmann, Space Sci. Rev. 80, 217 (1997).

[3] P. Song et al., Geophys. Monogr. 133, 121 (2003).

[4] A. Hasegawa, J. Geophys. Res. 81, 5083 (1976).

[5] L. Rezeau et al., J. Geophys. Res. 94, 101 (1989).

[6] A. Hasegawa and K. Mima, J. Geophys. Res. 83, 1117 (1978).

[7] B. U. O. Sonnerup, J. Geophys. Res. 85, 2017 (1980).

[8] W. Lotko and B. U. O. Sonnerup, Geophys. Monogr. 90, 371 (1995).

[9] J. R. Johnson and C.Z. Cheng, Geophys. Res. Lett. 24, 1423 (1997).

[10] J. A. Miller and D. A. Roberts, Astrophys. J. 452, 912 (1995).

[11] O. A. Kuznetsov et al., Astrophys. J. 514, 691 (1999).

[12] M. N. Rosenbluth, Plasma Phys. Controlled Fusion 41, A99 (1999).

[13] C.P. Escoubet et al., Space Sci. Rev. 79, 11 (1997).

[14] C. T. Russell, Cosmic. Electrodyn. 2, 184 (1971).

[15] A. Hasegawa et al., Nature (London) 430, 755 (2004).

[16] M. N. Nishino et al., Ann. Geophys. 25, 769 (2007).

[17] F. S. Mozer et al., Geophys. Res. Lett. 32, L24 102 (2005).

[18] C. C. Chaston et al., Phys. Rev. Lett. 95, 065002 (2005).

[19] L. Chen and A. Hasegawa, J. Geophys. Res. 79, 1033 (1974).

[20] J. R. Johnson, C.Z. Cheng, and P. Song, Geophys. Res. Lett. 28, 227 (2001).

[21] C. C. Chaston et al., J. Geophys. Res. 110, A02 211 (2005).

[22] H. J. Singer et al., J. Geophys. Res. 86, 4589 (1981).

[23] N. A. Tsyganenko, J. Geophys. Res. 107, A8 (2002).

[24] World Data Center for Geomagnetism, Definition of the Dst index, http://swdcdb.kugi.kyoto-u.ac.jp, Kyoto, Japan (2004).

[25] R. A. Treumann, J. LaBelle, and T. M. Bauer, Geophysical monograph 90, 331 (1995).

[26] L. Chen et al., Phys. Plasmas 8, 4713 (2001).

[27] J. R. Johnson and C.Z. Cheng, Geophys. Res. Lett. 28, 4421 (2001). 